\title{
Lidil
}

Revue de linguistique et de didactique des langues

$64 \mid 2021$

Le passif dans la langue parlée

\section{Salah Mejri, Luis Meneses-Lerin et Brigitte Buffard- Moret (dir.), La phraséologie française en questions}

Paris, Hermann, Vertige de la langue, 2020, 458 p.

\section{Alexis Ladreyt}

\section{OpenEdition}

\section{Journals}

Édition électronique

URL : https://journals.openedition.org/lidil/9308

DOI : $10.4000 /$ lidil.9308

ISSN : 1960-6052

\section{Éditeur}

UGA Éditions/Université Grenoble Alpes

Édition imprimée

ISBN : 978-2-37747-315-1

ISSN : $1146-6480$

\section{Référence électronique}

Alexis Ladreyt, « Salah Mejri, Luis Meneses-Lerin et Brigitte Buffard-Moret (dir.), La phraséologie

française en questions », Lidil [En ligne], 64 | 2021, mis en ligne le 01 novembre 2021, consulté le 24 novembre 2021. URL : http://journals.openedition.org/lidil/9308; DOI : https://doi.org/10.4000/lidil. 9308

Ce document a été généré automatiquement le 24 novembre 2021.

(C) Lidil 


\title{
Salah Mejri, Luis Meneses-Lerin et Brigitte Buffard-Moret (dir.), La phraséologie française en questions
}

\author{
Paris, Hermann, Vertige de la langue, 2020, 458 p.
}

\section{Alexis Ladreyt}

\section{RÉFÉRENCE}

Salah Mejri, Luis Meneses-Lerin et Brigitte Buffard-Moret (dir.), La phraséologie française en questions, Paris, Hermann, Vertige de la langue, 2020, $458 \mathrm{p}$.

1 La phraséologie, qui étudie les structures linguistiques préfabriquées du langage sous toutes leurs formes (collocation, dictons, parémies, etc.), est un domaine d'étude qui semble susciter un intérêt croisant dans la communauté des chercheurs. C'est dans ce contexte d'effervescence scientifique que le présent ouvrage a pris forme, lors de la deuxième rencontre internationale sur la phraséologie française organisée par l'Université d'Artois les 20 et 21 septembre 2017. Cet ouvrage est une collection de 26 contributions se répartissant en trois sous-domaines qui couvrent un large spectre applicatif de la phraséologie : la théorie et la description, les usages pragmatiques et appliqués et enfin les applications en didactique et en enseignement des langues. Il dresse un état des lieux à la fois diversifié et critique du champ de la phraséologie afin de questionner les principes épistémologiques et méthodologiques de la discipline au regard des avancées actuelles dans le domaine. Sans rentrer dans les détails des 26 contributions, toutes de très grande qualité, nous nous contenterons ici de citer / traiter / évoquer trois points centraux développés en filigrane tout au long de cet ouvrage : les critères définitoires, le statut linguistique et la dénomination, et enfin les applications et les méthodologies d'exploration.

2 Qu'est-ce qu'un phraséologisme? Comment peut-on le définir? Sur quels critères empiriques et opérationnels peut-on le catégoriser? C'est en proie à ces différents 
questionnements que Mejri, reconnu notamment pour ses travaux fondateurs sur le figement, introduit et replace les enjeux de cet ouvrage collectif: redessiner les contours méthodologiques et épistémologiques tout en prenant en compte les récentes extensions au domaine de la phraséologie. Ce fil d'Ariane épistémologique se retrouve tout au long des 26 articles qui, chacun à sa manière, revisitent, dépoussièrent, améliorent des critères définitoires des phraséologismes jusque lors considérés comme pérennes. Parmi ces critères revisités, la compositionnalité sémantique (le fait que l'addition des sens des unités qui composent la séquence phraséologique suffise ou non à exprimer le sens de la totalité de la séquence), l'opacité sémantique (ou idiomaticité selon les terminologies) et le figement morphosyntaxique, sémantique et pragmatique sont abordés. Ainsi, nous apprenons tout au long des 26 contributions que ces critères, bien que vérifiables sur une large proportion des séquences appartenant à la classe des phraséologismes, ne suffisent plus à définir une catégorie stable et unifiée. De même, le critère de polylexicalité qui a longtemps été considéré comme un critère stable de la catégorie ne semble plus tout à fait pertinent aux vues d'objets phraséologiques tels que les Actes de langages stéréotypés ou ALS (Kauffer, ce volume) ou les pragmatèmes (Mel'cuk, 2013) au sein desquels on peut retrouver des phraséologismes tels que chapeau! ou chiche!

Qui dit diversité des types phraséologiques, dit aussi diversité des statuts linguistiques et des dénominations. Et c'est bien là le second problème soulevé dans la plupart des contributions, l'inflation terminologique qui règne au sein du champ disciplinaire, et l'accroissement récent des sous-types phraséologiques qui sont parfois soumis à un nombre tellement élevé de contraintes que la catégorie ne devient plus opérable. L'ouvrage montrera ainsi tout au long des contributions qu'il est possible de répondre à cet enjeu par des catégories plus englobantes et opérationnelles, ce que DiazRodriguez ou Colson montreront dans leur contribution. D'autres contributions, parmi lesquelles celles de Mizouri, de Lajmi et de Hosni, nous montrent que de nouvelles extensions sont possibles et permettent de donner un éclairage très intéressant sur de nouvelles perspectives quant au statut linguistique du phraséologisme. Celui-ci n'est pas forcément une séquence indivisible et suffisante à elle-même, mais peut être dotée d'une structure concaténée complexe comme il peut concourir à la cohésion textuelle ou interactionnelle du contenu linguistique.

L'ouvrage explore également une multitude de champs applicatifs parmi lesquels la didactique, la linguistique de corpus ou le traitement automatique des langues. Les contributions de Burrows et Cetro, de Kodabocus et Masset-Martin ainsi que de Krylyschin nous montrent que la sensibilisation aux caractéristiques formelles des unités phraséologiques et à leurs spécificités d'emploi a, que ce soit en français langue étrangère, en français sur objectif universitaire ou en français langue de spécialité, un impact fort sur la mémorisation et l'acquisition de ces unités, créant ainsi une dynamique d'apprentissage de type [comprendre $\rightarrow$ manipuler $\rightarrow$ acquérir $\rightarrow$ réutiliser]. Avec les contributions de Blanco qui montre toute l'importance de l'approche diachronique pour la compréhension des dynamiques linguistiques anciennes fondant les emplois d'aujourd'hui, et de Gonon, Goossens et Novakova qui révèle tout le potentiel de l'analyse des constructions lexico-syntaxiques propre à des motifs récurrents dans le but de caractériser des sous-genres littéraires, l'ouvrage donne au lecteur une très grande diversité des approches et méthodologies d'exploration fonctionnelles et attestées sur corpus. Enfin, loin de se limiter au français, certaines des contributions parmi lesquelles celles de Bogacki (polonais), 
Palma (espagnol) et Rădulescu (roumain) envisagent la phraséologie dans une perspective contrastive qui ouvre de nombreuses possibilités dans la caractérisation des spécificités des phénomènes phraséologiques.

5 En conclusion, loin d'être un ouvrage exhaustif sur le domaine, La phraséologie française en questions propose avant tout une réflexion épistémologique et méthodologique de qualité sur la phraséologie. Bien que destiné principalement à un public scientifique averti, cet ouvrage s'avère tout aussi éclairant pour les débutants souhaitant s'aventurer dans ce champ de spécialité, pourvu qu'ils soient initiés à la terminologie de base. Il est évident que ces 26 contributions ne permettront pas de résoudre les problèmes théoriques évoqués, mais elles proposent en tout cas des pistes prometteuses et très stimulantes qui contribuent, nous en sommes convaincu, à la diversité et la vivacité scientifique de ce champ d'études.

\section{AUTEURS}

\section{ALEXIS LADREYT}

Univ. Grenoble Alpes, LIDILEM, 38000 Grenoble, France 